\title{
EL PAPEL DEL EJÉRCITO NACIONAL DE COLOMBIA PARA EL FORTALECIMIENTO DE LA PAZ EN EL POSACUERDO CON LAS FARC
}

Andrés Eduardo Fernández Osorio ${ }^{1}$

\begin{abstract}
Resumen. Con el propósito de establecer la naturaleza de las transformaciones militares realizadas usualmente en un escenario de posacuerdo, este estudio explora las experiencias de diez países con procesos de paz similares al que realizó el Gobierno colombiano con las Fuerzas Armadas Revolucionarias de Colombia (FARC) en noviembre de 2016. Utilizando una metodología de las ciencias militares denominada DOMPILEM y los datos de la Matriz de Acuerdos de Paz de la Universidad de Notre Dame, se analizan los componentes de capacidad militar con el fin de generar lecciones aprendidas útiles para el caso colombiano. Se concluye que la capacidad estatal es fundamental para garantizar la estabilidad e implementación del proceso de paz, escenario en el cual la acción unificada y unas relaciones civiles-militares sólidas son esenciales para garantizar el cumplimiento de la misión constitucional del Ejército Nacional de Colombia y soportar futuras iniciativas de reconciliación.
\end{abstract}

Palabras clave: acuerdo de paz; Colombia; Ejercito Nacional; posacuerdo; transformación militar.

\section{Introducción}

Tradicionalmente, los acuerdos de paz suscritos para terminar un conflicto tienden a reestructurar las Fuerzas Militares (FF. MM.) de un Estado para hacer frente a nuevos desafíos de seguridad y defensa. Asimismo, se tiende a utilizar parte del presupuesto usualmente asignado a las FF. MM. para cumplir con los

1 Teniente Coronel del Ejército Nacional de Colombia. Doctorando en Derecho y Ciencias Políticas (Universidad de Barcelona). Magíster en Economía, Estado y Sociedad: Política y Seguridad (Universidad Colegio de Londres, 2014). Magíster en Relaciones Internacionales (Escuela Superior de Economía de Moscú, 2014). Jefe de Investigación, Desarrollo Tecnológico e Innovación, Academia Militar de Colombia (Escuela Militar de Cadetes "General José María Córdova"). Contacto: andres.fernandez@esmic.edu.co; ORDIC: https://orcid.org/0000-0003-0643-0258. 
compromisos acordados. Sin embargo, reformas militares inadecuadas y recortes presupuestales desacertados pueden afectar la capacidad de un Estado para apoyar la consolidación de la paz y contrarrestar las amenazas persistentes. Por ejemplo, la reaparición de la violencia en países como el Congo, Guatemala, El Salvador, Líbano, Camboya, Ruanda, Burundi, Sudán y Liberia es asociada por diversos autores a la falta de una política apropiada de seguridad y defensa, así como a una inadecuada trasformación de sus FF. MM. (Holiday \& Stanley, 1993; Bourgois, 2001; Frieden, 2002; Roeder \&Rothcild, 2005; Autesserre, 2010; Ginty, 2010; Joshi, Melander \& Quinn, 2017; Joshi \& Wallensteen, 2018).

El caso colombiano puede no ser diferente. Desde la firma del acuerdo de paz con las Fuerzas Armadas Revolucionarias de Colombia (FARC) en noviembre de 2016, denominado oficialmente "Acuerdo final para la terminación del conflicto y la construcción de una paz estable y duradera", un número creciente de sectores han destacado los múltiples beneficios que representa el final del conflicto con uno de los grupos generadores de violencia en Colombia. No obstante, diversas complicaciones en su implementación han resultado en recriminaciones mutuas entre el Gobierno colombiano y las FARC, lo cual ha generado una inestabilidad de todo lo pactado e incluso ha hecho posible el resurgimiento de la violencia (Aya, 2017; Moreno, 2017; Iglesias \& Jiménez, 2018; Vacas, 2018; Vargas, 2018). De igual forma, esta situación ha contribuido al inicio de una discusión sobre la capacidad estatal para cumplir las obligaciones pactadas y sobre el papel del Ejército Nacional de Colombia en el fortalecimiento de la paz en el posacuerdo con las FARC.

A pesar de la relevancia de este tema, hay una cantidad limitada de estudios que han analizado técnicamente los retos que enfrenta el Ejército Nacional de Colombia para apoyar la implementación. Este documento busca ofrecer algunas perspectivas en este debate, al determinar cuáles han sido las lecciones aprendidas de países con acuerdos de paz similares al colombiano en la transformación de sus ejércitos en el escenario de posacuerdo. Para tal fin se realiza un análisis comparativo de los datos de la matriz de acuerdos de paz de la Universidad de Notre Dame a la luz de una metodología de las ciencias militares denominada DOMPILEM².

Los hallazgos de este estudio sugieren que Colombia necesita enfoques innovadores para el escenario del posacuerdo, en el cual la acción unificada y las relaciones cívico-militares deben desempeñar un papel fundamental. Del mismo modo, se encontró que si bien el Ejército Nacional de Colombia puede proporcionar asistencia crucial para la construcción de paz, debe continuar su proceso actual de

2 Dompilem: Doctrina, Organización, Material y Equipo, Personal, Infraestructura, Liderazgo, Educación y Mantenimiento; es la traducción de la sigla Dotmlpf en idioma inglés. 
transformación ${ }^{3}$ para garantizar su misión constitucional y soportar futuras iniciativas de reconciliación.

\section{Contexto del posacuerdo en Colombia}

En las últimas décadas, la literatura académica sobre análisis de conflictos y consolidación de procesos de paz ha crecido considerablemente, además se destaca que en este campo es central el estudio de la capacidad estatal ${ }^{1}$ para apoyar la implementación de dichos compromisos. Por ejemplo, Paris (2004) y Rocha (2011) enfatizan la necesidad de que se realice una reconstrucción eficiente de las instituciones estatales y el estado de derecho para fomentar un escenario estable de posacuerdo. Asimismo, otros estudios sugieren que la capacidad estatal "puede mejorar la paz y minimizar la recurrencia de conflictos" (Bercovitch \& DeRouen, 2008, p. 70) y que los Estados con instituciones sólidas tienen "menos probabilidades de experimentar el inicio de una guerra civil [...] porque responderán a las necesidades de sus ciudadanos y son conscientes del hecho de que los problemas más allá de los estrictamente militares, juegan un papel debilitante en la vida cotidiana" (Taydas, Peksen \& James, 2010, p. 196).

Como lo sugieren Walter (1999) y Hartzell (2010), la fase de implementación de un acuerdo de paz es el periodo más inestable, de manera que es fundamental reorganizar las prioridades de las políticas públicas y las instituciones responsables de apoyar el cumplimiento de los compromisos. Así las cosas, la capacidad estatal es esencial cuando el acuerdo de paz se desarrolla en un escenario inestable con otros actores generadores de violencia y hay recursos muy limitados para su implementación.

Este es precisamente la situación que vive Colombia. El acuerdo de paz de 2016 se resolvió con un solo actor armado, las FARC, pero otros Grupos Armados Organizados $(\mathrm{GAO})^{5}$ como el Ejército de Liberación Nacional (ELN), el Ejército Popular de Liberación (EPL) y las disidencias de las FARC mantienen cierto predominio en algunas zonas del territorio colombiano y han comenzado a competir por

3 Este artículo define la transformación militar como el proceso continuo de innovación que implica la preparación adecuada y oportuna de una fuerza militar para enfrentar futuras amenazas y desafíos.

4 Este artículo define la capacidad del Estado como la habilidad para diseñar y aplicar satisfactoriamente las políticas públicas.

5 Definidos por el Ministerio de Defensa de Colombia (2016) como grupos que utilizan la violencia en contra de las Fuerzas Armadas o de otras instituciones del Estado, la población civil, la propiedad civil u otros grupos armados, con la capacidad de generar niveles de violencia armada que superan la de disturbios y tensiones internas, con organización, comando responsable y control sobre una parte del territorio. 
las antiguas áreas controladas por las FARC (Álvarez, 2016; Ávila, 2016). Además, la implementación del acuerdo, con un costo estimado de 130 mil millones de pesos colombianos por los próximos quince años (Sáenz, 2017), requerirá alternativas innovadoras para reforzar la capacidad estatal debido a la reducción de la financiación internacional, una economía colombiana volátil que depende en gran medida de los precios fluctuantes del petróleo y del pago de una deuda externa cercana al $40 \%$ del Producto Interno Bruto (Castańo, 2017; Dinero, 2018).

A pesar de este complicado escenario, en el cual deben usarse todas las competencias del Estado, especialmente en el contexto de defensa y seguridad, poco se ha discutido sobre las capacidades del Ejército Nacional para contribuir al fortalecimiento de la paz en un escenario de posacuerdo. Eventos recientes como las dificultades del Gobierno colombiano para construir la infraestructura acordada para desmovilizar a los combatientes de las FARC (Escobar, 2017; Semana, 2017); los problemas de la Organización de las Naciones Unidas (ONU) para ubicar los contenedores de almacenamiento con las armas decomisadas de las FARC (Gómez, 2017) y el aumento de casi $42 \%$ en el cultivo de coca y $60 \%$ en la producción de cocaína durante los últimos tres años (El País, 2017; El Nuevo Herald, 2017) representan un riesgo para la totalidad del acuerdo y corroboran el debate sobre la participación del Ejército colombiano en la construcción de la paz.

\section{Metodología}

Para identificar lecciones aprendidas de la transformación de los ejércitos en escenarios de posacuerdo, se identificaron en primer lugar las características del proceso de paz colombiano con las FARC y, a partir de estas, se determinaron posteriormente los países con procesos de paz similares al colombiano y que realizaron reformas a sus ejércitos.

Si bien estudios previos han utilizado diferentes métodos para evaluar el proceso de paz en Colombia (Díaz, Fernández \& Rodríguez, 2013; Meacham, Farah \& Lamb, 2014; Molano, 2015), en esta investigación se eligió hacer un análisis comparativo debido a que el marco teórico ofrecido por la Matriz de Acuerdos de $\mathrm{Paz}$ (en adelante, MAP), del Instituto Kroc para Estudios Internacionales de Paz en la Universidad de Notre Dame ${ }^{6}$, permite identificar con facilidad los contextos correspondientes. La MAP clasifica 34 acuerdos de paz en 32 países entre 1989 y

6 Disponible en https://peaceaccords.nd.edu/ 


\section{2 según 51 tipos de disposiciones, lo cual hace posible caracterizar el contenido de un acuerdo y sus documentos soportes (tablas 1 y 2 ).}

Tabla 1. Disposiciones de la Matriz de Acuerdos de Paz (MAP) del Instituto Kroc para Estudios Internacionales de $\operatorname{Paz}(N=51)$

\begin{tabular}{|c|c|c|}
\hline Amnistía / Perdón & $\begin{array}{l}\text { Descentralización / } \\
\text { federalismo }\end{array}$ & Reforma constitucional \\
\hline Apoyo de donantes & Desmovilización & Reforma de ciudadanía \\
\hline Arbitraje internacional & Embargo de armas & $\begin{array}{l}\text { Reforma } \\
\text { de la administración civil }\end{array}$ \\
\hline $\begin{array}{l}\text { Autoridad transitoria } \\
\text { de la ONU }\end{array}$ & $\begin{array}{l}\text { Fuerza de paz de las Naciones } \\
\text { Unidas }\end{array}$ & $\begin{array}{l}\text { Reforma de la rama } \\
\text { legislativa }\end{array}$ \\
\hline Cese al fuego & $\begin{array}{l}\text { Fuerza regional } \\
\text { de mantenimiento de paz }\end{array}$ & $\begin{array}{l}\text { Reforma de los medios } \\
\text { de comunicación }\end{array}$ \\
\hline $\begin{array}{l}\text { Comisión para abordar daños / } \\
\text { pérdidas }\end{array}$ & $\begin{array}{l}\text { Gobierno de transición / poder } \\
\text { compartido }\end{array}$ & Reforma del poder ejecutivo \\
\hline $\begin{array}{l}\text { Comité de resolución } \\
\text { de disputas }\end{array}$ & Grupos paramilitares & Reforma educativa \\
\hline $\begin{array}{l}\text { Cronograma detallado } \\
\text { de implementación }\end{array}$ & Lenguaje oficial y símbolo & $\begin{array}{l}\text { Reforma electoral/ } \\
\text { partidos políticos }\end{array}$ \\
\hline Demarcación de límites & Liberación de prisioneros & Reforma judicial \\
\hline $\begin{array}{l}\text { Derecho } \\
\text { de autodeterminación }\end{array}$ & Manejo de recursos naturales & Reforma militar \\
\hline Derechos de las minorías & Mecanismo de ratificación & Reforma policial \\
\hline $\begin{array}{l}\text { Derechos de las minorías } \\
\text { indígenas }\end{array}$ & $\begin{array}{l}\text { Mecanismo de verdad o recon- } \\
\text { ciliación }\end{array}$ & Refugiados \\
\hline Derechos de las mujeres & $\begin{array}{l}\text { Mecanismo de verificación/ } \\
\text { monitoreo }\end{array}$ & Reintegración \\
\hline Derechos de los niños & $\begin{array}{l}\text { Personas desplazadas } \\
\text { internamente }\end{array}$ & Relaciones interétnicas / Estado \\
\hline Derechos humanos & Poder territorial compartido & Reparación / Indemnización \\
\hline Desarme & Protecciones culturales & Retiro de tropas \\
\hline $\begin{array}{l}\text { Desarrollo económico } \\
\text { y social }\end{array}$ & $\begin{array}{l}\text { Referéndum } \\
\text { de independencia }\end{array}$ & Revisión del acuerdo \\
\hline
\end{tabular}

Fuente: Elaborada por el autor con base en la información de Kroc (2018). 
Tabla 2. Países analizados en la Matriz de Acuerdos de Paz (MAP) del Instituto Kroc para Estudios Internacionales de Paz $(N=32)$

\begin{tabular}{llll}
\hline Angola & Yibuti & Costa de Marfil & Irlanda del Norte \\
Bangladesh & Timor oriental & Líbano & Papúa Nueva Guinea \\
Bosnia y Herzegovina & El Salvador & Liberia & Ruanda \\
Burundi & Filipinas & Macedonia & Senegal \\
Camboya & Guatemala & Mali & Sierra Leona \\
Colombia & Guinea-Bisáu & Mozambique & Sudáfrica \\
Congo & India & Nepal & Sudan \\
Croacia & Indonesia & Níger & Tayikistán \\
\hline
\end{tabular}

Fuente: Elaborada por el autor con base en la información de Kroc (2018).

Dado el marco de referencia de la MAP, se realizó un examen detallado del documento final del acuerdo de paz del 24 de noviembre de 2016 en Colombia ${ }^{7}$. Este ejercicio permitió tener una mejor comprensión del contenido del acuerdo y caracterizar su contenido con base en las 51 disposiciones de la MAP, de manera que se logró identificar 35 disposiciones establecidas en la Matriz que están incluidas en el acuerdo de paz colombiano (tabla 3).

Tabla 3. Disposiciones de la Matriz de Acuerdos de Paz (MAP) incluidas en el acuerdo de paz con las FARC $(N=35)$

\begin{tabular}{|c|c|c|}
\hline Amnistía & Derechos de los niños & Protecciones culturales \\
\hline Apoyo de donantes & Derechos humanos & Reforma constitucional \\
\hline Arbitraje internacional & Desarme & $\begin{array}{l}\text { Reforma } \\
\text { de la administración civil }\end{array}$ \\
\hline $\begin{array}{l}\text { Autoridad transitoria } \\
\text { de la ONU }\end{array}$ & Desarrollo económico y social & Reforma de la rama legislativa \\
\hline Cese al fuego & Desmovilización & $\begin{array}{l}\text { Reforma de los medios } \\
\text { de comunicación }\end{array}$ \\
\hline $\begin{array}{l}\text { Comisión para abordar daños/ } \\
\text { pérdidas }\end{array}$ & Grupos paramilitares & Reforma educativa \\
\hline
\end{tabular}

7 Disponible en http://www.altocomisionadoparalapaz.gov.co/procesos-y-conversaciones/Documentos\%20 compartidos/24-11-2016NuevoAcuerdoFinal.pdf 


\begin{tabular}{|c|c|c|}
\hline $\begin{array}{l}\text { Comité de resolución } \\
\text { de disputas }\end{array}$ & Liberación de prisioneros & $\begin{array}{l}\text { Reforma electoral/ } \\
\text { partidos políticos }\end{array}$ \\
\hline $\begin{array}{l}\text { Cronograma detallado } \\
\text { de implementación }\end{array}$ & Manejo de recursos naturales & Reforma judicial \\
\hline Derecho de autodeterminación & Mecanismo de ratificación & Reforma militar \\
\hline Derechos de las minorías & $\begin{array}{l}\text { Mecanismo de verdad } \\
\text { o reconciliación }\end{array}$ & Refugiados \\
\hline $\begin{array}{l}\text { Derechos de las minorías } \\
\text { indígenas }\end{array}$ & $\begin{array}{l}\text { Mecanismo de verificación/ } \\
\text { monitoreo }\end{array}$ & Reintegración \\
\hline Derechos de las mujeres & $\begin{array}{l}\text { Personas desplazadas } \\
\text { internamente }\end{array}$ & Reparación / Indemnización \\
\hline
\end{tabular}

Fuente: Elaborada por el autor con base en la información del Alto Comisionado para la Paz (2016) y Kroc (2018).

Como se esperaba, estas disposiciones incluyen algunos de los objetivos más importantes logrados, como el cese al fuego, el desarme y las reparaciones. La inclusión de estos temas en el acuerdo se puede entender como signo de una comprensión común de la necesidad urgente que tiene Colombia de resolver el conflicto, de las expectativas de la comunidad internacional y de la responsabilidad necesaria para las acciones y los resultados del conflicto.

A continuación, se determinaron los países incluidos en la MAP que contuvieran la mayor cantidad de disposiciones en sus procesos de paz similares al acuerdo colombiano y que, a su vez, incluyeran reformas militares como parte de sus compromisos. Como resultado se encontraron 24 países con ocurrencias de entre 8 y 29 disposiciones utilizadas también por Colombia. Con el objetivo de optimizar la muestra, se calculó la mediana de las ocurrencias para identificar los países que comparten en una mayor proporción (mediana $=18$ ) las mismas disposiciones que Colombia en sus acuerdos de paz. De este filtro se obtuvieron diez países con los cuales se desarrolló el resto del estudio: Burundi ${ }^{8}$, El Salvador ${ }^{9}$, Guatemala $^{10}$,

8 El Acuerdo de Paz y Reconciliación de Arusha para Burundi, firmado el 28 de agosto de 2000.

9 El Acuerdo de Paz de Chapultepec, firmado el 16 de enero de 1992.

10 El Acuerdo por una Paz Firme y Duradera, firmado el 29 de diciembre de 1996. 
Indonesia $^{11}$ Liberia $^{12}$, Mozambique ${ }^{13}$, Nepal $^{14}$, Ruanda ${ }^{15}$, Sierra Leona ${ }^{16}$ y Sudán ${ }^{17}$ (tabla 4). Esta selección no pretende implicar que los procesos de paz y sus particularidades son iguales; solo sugiere que el discurso teórico de los casos elegidos es similar al proceso de paz colombiano y, en consecuencia, puede servir como un marco de referencia para analizar las perspectivas del acuerdo de paz colombiano.

\section{Transformaciones militares en escenarios de posacuerdo}

Para determinar las lecciones aprendidas de las transformaciones a las Fuerzas Militares que definieron los diez países identificados en los escenarios de posacuerdo, se estudiaron las reformas de los militares en los diez casos utilizando la base de datos de los acuerdos de paz de la ONU ${ }^{18}$ a través de la metodología DOMPILEM. Como se había mencionado, esta metodología analiza la Doctrina, Organización, Material (equipamiento militar), Personal, Infraestructura, Liderazgo, Educación y Mantenimiento involucrados en un proyecto militar, con el objetivo de identificar las debilidades, oportunidades, fortalezas y amenazas inherentes, así como de proporcionar una estimación de la fiabilidad de su alcance y métodos (FernándezOsorio, 2016). Los resultados del análisis son los siguientes:

\section{Doctrina}

Una práctica común observada en los diez casos es la asignación de nuevas funciones para las Fuerzas Militares y, por lo tanto, el diseño de una nueva doctrina militar para respaldar tales responsabilidades. Las nuevas tareas acordadas imponen generalmente una restricción al uso de la fuerza y encaminan las capacidades militares principalmente en la defensa de la soberanía nacional y la integridad territorial. El acuerdo de Burundi es tal vez el más completo al momento de describir los

11 El Memorando de Entendimiento entre el Gobierno de la República de Indonesia y el Movimiento Aceh Libre, firmado el 15 de agosto, 2005.

12 El Acuerdo de Paz de Accra, firmado el 18 de agosto de 2003.

13 El Acordo Geral de Paz (AGP), firmado el 4 de octubre de 1992.

14 El Acuerdo General de Paz, firmado el 21 de noviembre de 2006.

15 Los Acuerdos de Arusha, firmados el 4 de agosto de 1993.

16 El Acuerdo de Paz de Lomé, firmado el 7 de julio de 1999.

17 El Acuerdo de Paz Integral Sudán, firmado el 9 de enero de 2005.

18 Disponible en http://peacemaker.un.org/document-search. 


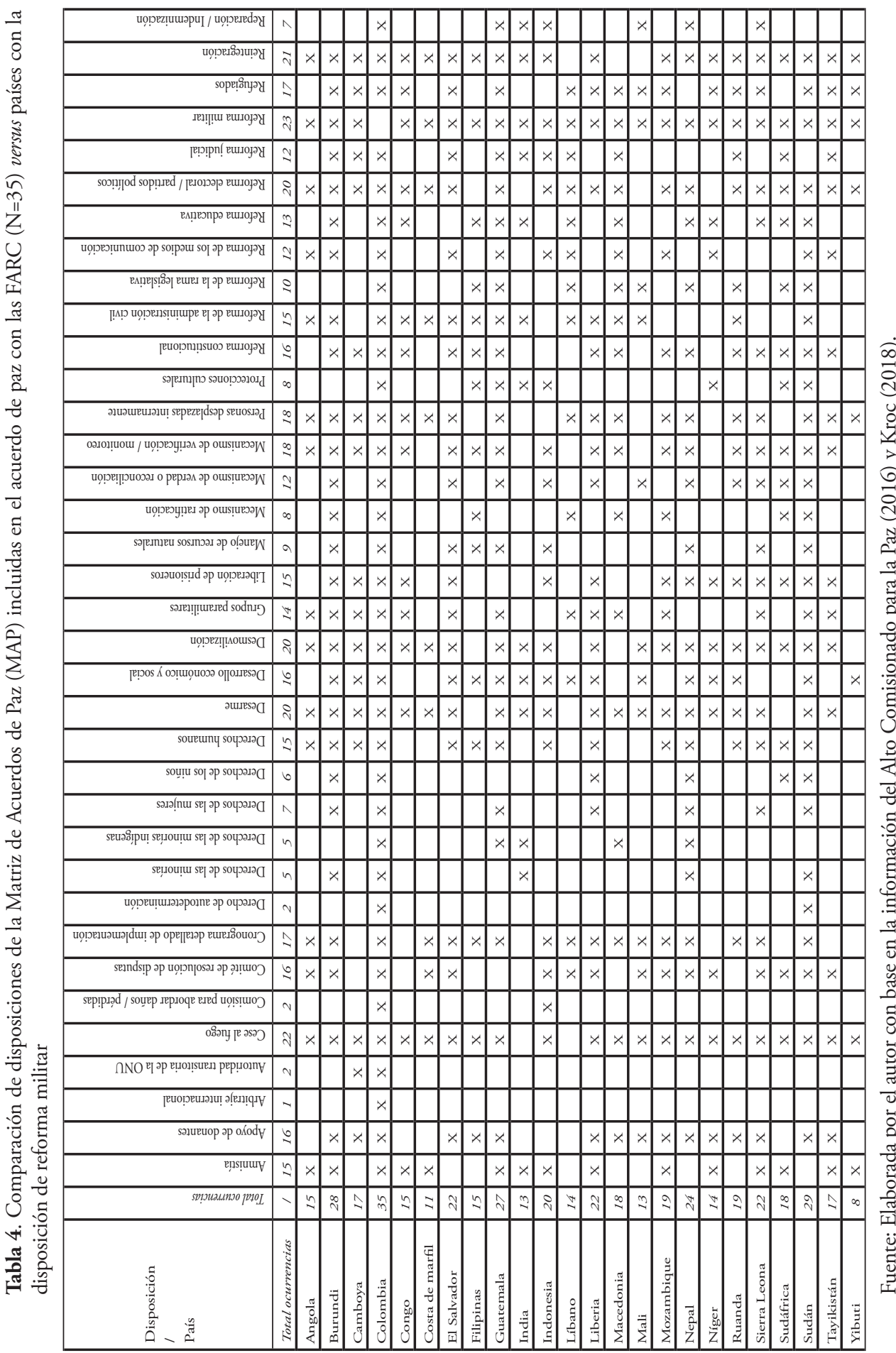


nuevos papeles y misiones asignados al Ejército, además reconoce la posibilidad de usarlo para apoyar la consolidación de la paz; por el contrario, el acuerdo de paz de Indonesia desconoce esa posibilidad, restringiendo al Ejército a la defensa externa.

Por su parte, el acuerdo de paz de El Salvador es innovador, ya que enfatiza cinco características que describen el papel del Ejército: conscripción, movilización y reservas, organizaciones paramilitares, servicios de seguridad privada y una comisión de la verdad posacuerdo. Al definir la naturaleza del servicio militar obligatorio, El Salvador estableció un marco para el empleo de las reservas y la necesidad de servicios de seguridad privados, con lo cual apoyó una visión weberiana del Estado en términos de garantizar el monopolio de la violencia para gobernar efectivamente.

Una desventaja importante de esas nuevas funciones asignadas a las Fuerzas Militares en el posacuerdo es la exclusión de los ejércitos de la consolidación de la paz, desconociendo sus capacidades, instalaciones, equipo y personal. Otras misiones posibles, como el apoyo a las operaciones en desastres nacionales, la asistencia a las autoridades civiles en el mantenimiento del orden público y la participación en operaciones internacionales de apoyo a la paz son apenas mencionadas.

\section{Organización}

En los casos seleccionados, se puede observar que las Fuerzas Militares de diversos escenarios de posacuerdo tienden a reestructurarse y modernizarse para hacer frente a sus nuevas tareas. Las funciones de defensa, seguridad e inteligencia son separadas por la creación o reorganización de organismos independientes, con el objetivo de maximizar la responsabilidad y la transparencia. La integración de excombatientes de todas las partes en las Fuerzas Militares es una práctica común, así como la exclusión del personal involucrado en violaciones de derechos humanos o crímenes de guerra; asimismo, las unidades de las Fuerzas Militares involucradas en transgresiones son disueltas y nuevas unidades conjuntas son organizadas combinando exmilitares y desmovilizados.

Se presta atención a la estructura de la cadena de mando, la obediencia a la autoridad civil y la legitimidad de las órdenes a través del presidente, como comandante en jefe de las Fuerzas Militares, y el ministro de Defensa. Del mismo modo, una parte importante de los civiles se ubican en puestos de asesoramiento en el Ministerio de Defensa o en el Ejército para proporcionar asesoramiento político-técnico y fundamentos para mejorar las relaciones cívico-militares. 
Al evaluar el contexto de los casos seleccionados, se puede observar una tendencia a considerar que la firma de un acuerdo de paz implica un escenario inmediato de estabilidad y terminación de la violencia, y que ya no se requiere que las Fuerzas Militares tengan la misma fortaleza. Esto lleva a la idea de que las capacidades militares deben ser reducidas y que una parte importante de los presupuestos de defensa debe ser utilizada para financiar la implementación del acuerdo. Sin embargo, a pesar de un proceso efectivo de Desarme, Desmovilización y Reintegración (DDR), en un escenario de posacuerdo es posible que surjan nuevas dinámicas de ilegalidad que pueden fomentar la reaparición de la violencia.

\section{Material (equipamiento militar) y mantenimiento}

Dos elementos se mencionan comúnmente en los casos analizados cuando se habla del equipamiento y mantenimiento del material (de guerra) de las Fuerzas Militares en un escenario de posacuerdo: 1) la política de seguridad y defensa, además de las posibles amenazas contempladas en esta, y 2) la estabilidad financiera del país y el presupuesto asignado a las Fuerzas Militares. El primer elemento determina el tipo de Fuerzas Militares requeridas por un Estado, mientras que el segundo define el gasto militar posible y, en consecuencia, la cantidad de material que se puede costear, así como su mantenimiento y ciclo de vida.

En solo cuatro países, Burundi, El Salvador, Guatemala y Sudán, se menciona una disminución en el gasto militar como parte de los acuerdos de paz; sin embargo, en todos los casos seleccionados se presenta una variación en la asignación de presupuesto a las Fuerzas Militares (figura 1). Al analizar el gasto militar versus un cronograma del conflicto desde cinco años antes del acuerdo de paz hasta diez años después, se puede identificar una reducción significativa de este, aun a pesar de la persistencia de amenazas a la seguridad y defensa (figura 2). Sorprendentemente, ninguno de los casos considera la mejora de una industria de defensa nacional como fuente de tecnología para promover el proceso de reconstrucción, impulsar la economía o apoyar el empleo. 


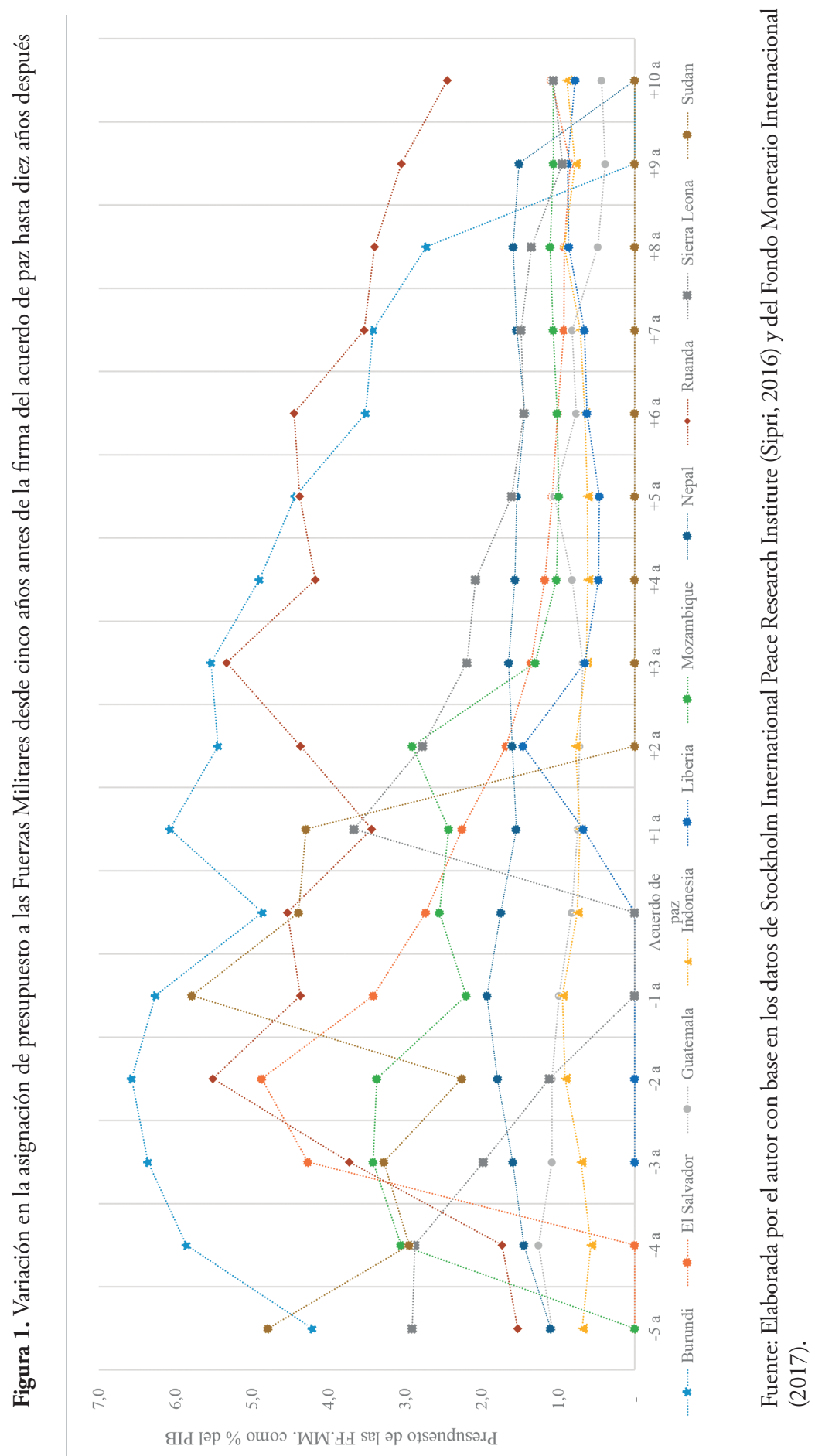




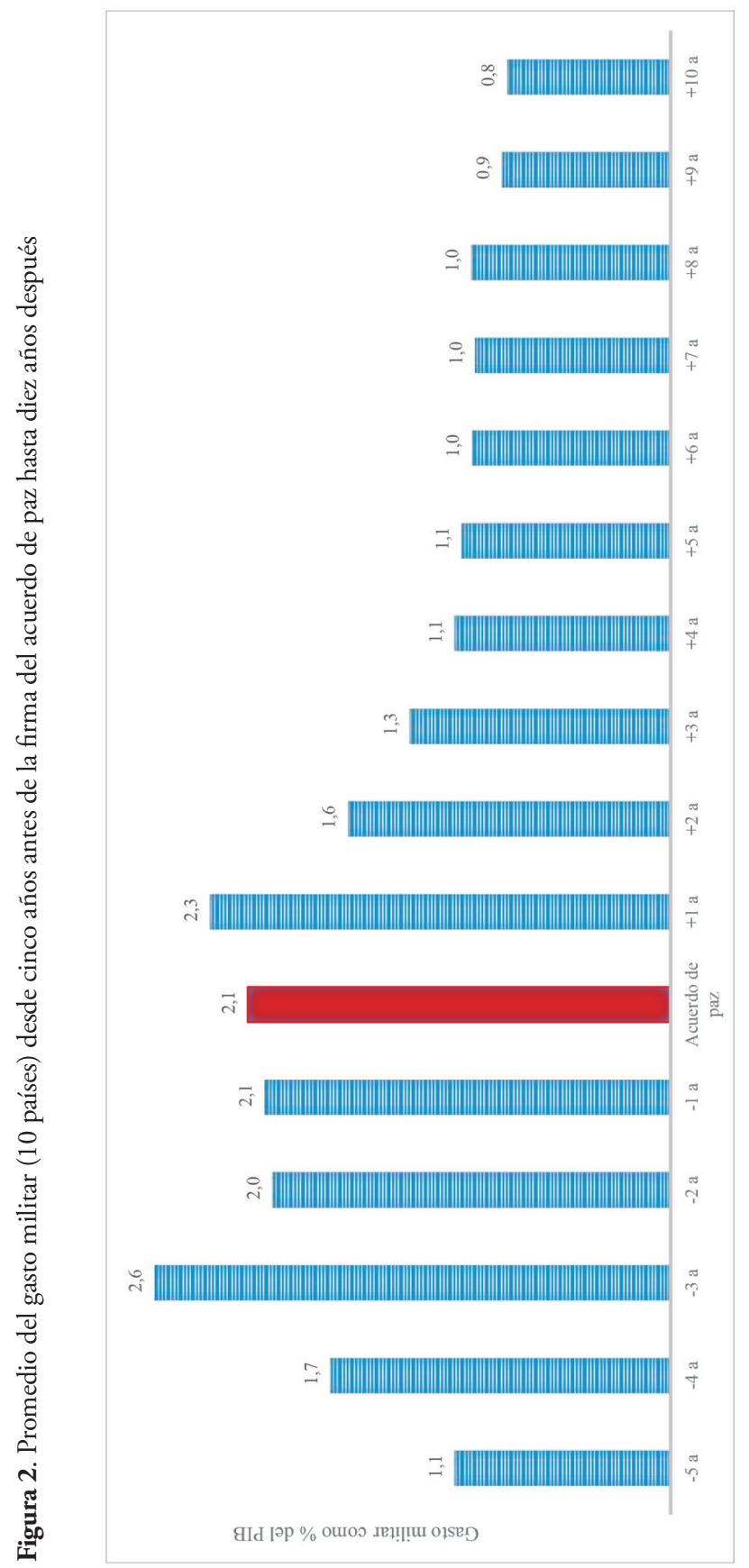

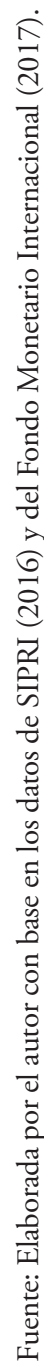




\section{Personal}

En los casos estudiados, generalmente se adoptan nuevas disposiciones sobre el personal que integra las Fuerzas Militares. Se fomenta la idea de un militar profesional, no partidista, no deliberativo y étnico-religiosamente equilibrado a garantizar la obediencia a la ley y la Constitución, la subordinación a la autoridad civil y el respeto de los derechos humanos. Los derechos fundamentales de los hombres y mujeres de las Fuerzas Militares se fortalecen, enfatizando la importancia de reconocer sus particularidades y privilegios individuales como ciudadanos; sin embargo, solo los acuerdos de Burundi y Ruanda consignaron el derecho a votar de los militares como parte del proceso de reconciliación.

A pesar de la necesidad de avanzar hacia ejércitos profesionales, pocas decisiones efectivas se tomaron para tal fin, ya que se apoyaron principalmente en el reclutamiento y el servicio militar obligatorio como la principal fuente de personal. Sin embargo, cabe mencionar que el personal con historial de violaciones de derechos humanos o delitos de lesa humanidad fueron separados como parte de un proceso de depuración obligatorio.

En este escenario es importante recordar que unas Fuerzas Militares modernas deben estar formadas idealmente por tropas convencidas de su papel en la sociedad y no por grandes cantidades de reclutas obligados a servir, generalmente en contra de su voluntad. Además, la conscripción priva al país de hombres y mujeres esenciales para apoyar un escenario de posacuerdo y sus desafíos financieros. Esto no implica que servir en las Fuerzas Militares no sea esencial para un Estado, pero algunas mentes y talentos pueden servir de una mejor forma en ocupaciones no militares que garanticen la construcción de una paz sostenible.

\section{Infraestructura}

Ninguno de los acuerdos de paz menciona expresamente la mejora de las instalaciones militares porque este es un problema técnico que resulta de la transformación militar. Sin embargo, en los casos en que se acordó una reducción del pie de fuerza y de bases militares, estas decisiones permitieron, a la larga, racionalizar el gasto y tomar mejores determinaciones sobre tropas y material no requerido a la luz de una nueva política de seguridad y defensa. La sólida integración de capacidades hacia fuerzas multimisión facilita el desmantelamiento de las instalaciones militares y la reorientación de la financiación hacia proyectos estratégicos productivos que pueden beneficiar a las Fuerzas Militares y ayudar en la implementación de las disposiciones acordadas. 


\section{Liderazgo}

Una práctica observada, especialmente en los casos africanos, es la redefinición de la cadena de mando y la integración de exrebeldes como oficiales de alta graduación para compartir el poder y la toma de decisiones. Del mismo modo, algunos de los antiguos líderes rebeldes se integraron en el liderazgo civil en las ramas legislativa, ejecutiva y judicial, con una categoría algunas veces superior a los oficiales militares de alto rango. Esta práctica también se realizó con los cuerpos de oficiales y suboficiales para formar unidades conjuntas o nuevas unidades dentro de las Fuerzas. Antiguos soldados y rebeldes se mezclaron para fomentar confianza entre las partes y las oportunidades para establecer Fuerzas Militares reestructuradas.

Aunque esta práctica ayudó a fortalecer los procesos de reconciliación entre los bandos y alentó la creación de lazos democráticos dentro de las instituciones, también afectó el alistamiento de las unidades militares. Un entendimiento erróneo de los combatientes irregulares y las tropas oficiales como iguales en términos de perfil profesional, motivación personal, compromiso de servicio, experiencia técnica y capacitación afectó al esprit de $\operatorname{corps}^{19}$ y la preparación para el combate, y, por lo tanto, la posibilidad de utilizar las capacidades militares a tiempo y de manera efectiva para cumplir con los objetivos tácticos, operativos y estratégicos.

Si bien ambos bandos, rebeldes y tropas estatales, pueden tener similitudes con respecto a su capacidad para usar armas, realizar tareas tácticas y liderar a las tropas en combate, es fundamental reconocer que el papel de un miembro de las Fuerzas Militares no se debe a su valor como combatiente, sino a la formación integral que ha recibido y que permite a los ciudadanos confiar en él la defensa del Estado. La falta de comprensión de este aspecto por parte de algunos legisladores llevó a la formación de unidades mixtas de exinsurgentes y exmiembros de las Fuerzas Militares que no cumplen adecuadamente su misión debido a una deficiencia de cohesión, propósito, disciplina y liderazgo.

El liderazgo adecuado es fundamental dentro de las Fuerzas Militares porque la obediencia puede hacerse cumplir, pero el respeto y la lealtad dependen en gran medida de la legitimidad de la cadena de mando y sus capacidades. Líderes militares excepcionales ayudan a mejorar las relaciones civiles-militares debilitadas, la obediencia a las instituciones civiles y hacen que tanto la sociedad en general como las Fuerzas Militares sean conscientes de la importancia de sus deberes y derechos; en resumen, aumenta la legitimidad y la capacidad estatal.

19 Definido como "el espíritu común que existe en los miembros de un grupo e inspira entusiasmo, devoción y fuerte consideración por el honor del grupo” (Merriam-Webster, 2017, s.v. esprit corps). 


\section{Entrenamiento}

En los casos estudiados se evidencia que el entrenamiento de las Fuerzas Militares en un escenario de posacuerdo se basa en modelos inclusivos que mezclan temas modernos y tradicionales, con el objetivo de proporcionar a los hombres y mujeres una visión integral de sus responsabilidades y su papel dentro de la sociedad. Las relaciones civiles-militares, los derechos humanos, la educación sociopolítica y una cultura de paz y moralidad, entre otros, se incluyen en los programas para enfatizar la importancia de un ejército educado que entienda su deber para con la nación y la confianza depositada en sus miembros.

Naturalmente, la preparación técnica desempeña un papel fundamental en la estandarización de los procedimientos y en la incorporación de combatientes irregulares a una carrera militar estándar; con ese fin, las instituciones de capacitación militar se reforman o son creadas para preparar a los militares en sus nuevas responsabilidades. Esta transición ha sido respaldada a menudo por equipos internacionales de mentores, especialmente en casos africanos, que han ayudado a crear programas educativos, carreras profesionales, equivalencias de grado y estatutos de carrera.

Aunque algo controvertido, un enfoque liberal dentro de la educación militar en temas como política, economía, historia, derecho y diplomacia, proporciona al personal de las Fuerzas Militares una comprensión como ciudadanos y funcionarios con responsabilidades que otorgan autoridad, pero no superioridad sobre la población. Esto fomenta la apertura democrática y facilita la eliminación de los privilegios que pueden considerarse ilegítimos y que hubieran sido creados durante el conflicto.

\section{Lecciones para un escenario de posacuerdo en Colombia}

El análisis de los diez casos proporciona información valiosa sobre las decisiones tomadas por los gobiernos para cumplir los acuerdos de paz, particularmente cuando su índice de implementación después de diez años está en promedio por encima del 80 \% (Kroc, 2018). Sin embargo, la singularidad de los casos muestra que sus lecciones aprendidas, aunque importantes, deben evaluarse a la luz de las circunstancias colombianas y el interés nacional, de modo que proporcionen perspectivas realizables para el fortalecimiento de la paz. En este sentido, Deas (2012, p. 2) argumenta que lograr un acuerdo de paz con las FARC "no disminuye la necesidad de mejoras en la seguridad, la justicia, la policía y la presencia militar: la experiencia muestra que la desmovilización puede aumentar la criminalidad". 
La implementación de las disposiciones de un acuerdo de paz implica una tarea colosal para los gobiernos actuales y futuros; la limitación del presupuesto nacional, el disminuido apoyo financiero de la comunidad internacional, la discordia popular por el supuesto tratamiento privilegiado dado a las FARC y el deterioro de la percepción de seguridad han comenzado a crear dificultades para implementar el acuerdo en su totalidad.

La comparación de las experiencias de los diez países demuestra la necesidad de diseñar una estrategia original para consolidar la paz, en la cual las Fuerzas Militares pueden tener un papel central en la formación de la capacidad estatal para aplicar las disposiciones acordadas en virtud de un cronograma exigente. En consecuencia, el Gobierno colombiano debe enfocar sus esfuerzos en cuatro áreas principales que pueden fortalecer la paz y mejorar la capacidad del Ejército Nacional para enfrentar las amenazas persistentes y los desafíos futuros.

En primer lugar, las relaciones cívico-militares deberían optimizarse para que los líderes nacionales y los responsables de la formulación de políticas asuman su responsabilidad natural en seguridad y defensa, al mismo tiempo que se enfatiza la función de los militares en la sociedad. Las iniciativas de seguridad y defensa han tenido diferentes perspectivas durante el conflicto de casi sesenta años, algunas de las cuales fueron incompatibles o contradictorias (Vargas, 2010; Patiño, 2013); un claro ejemplo de esto es la ausencia, hasta la fecha, de una ley nacional que establezca clara y permanentemente el papel y la misión de las Fuerzas Militares para optimizar sus procesos, mejorar la toma de decisiones e innovar en planificación estratégica.

En Colombia no se ha logrado entender a las Fuerzas Militares como asesoras en la formulación de política pública, sin alejarse de su condición como ciudadanos sujetos de derechos y deberes al igual que cualquier otro representante del Estado. Esto ha facilitado la creación de una división errónea entre militares y ciudadanos, así como la existencia de dos sociedades en el imaginario colectivo: la militar y la sociedad civil. Unas relaciones cívico-militares adecuadas pueden favorecer un acercamiento entre las Fuerzas Militares y la sociedad, lo cual fomentaría espacios para resolver agravios y promover la reconciliación entre los excombatientes actuales y futuros.

En segundo lugar, fortalecer la legitimidad del Gobierno y ganarse los corazones y las mentes de todos los ciudadanos debería ser una prioridad para difundir la credibilidad en el escenario de posacuerdo. La experiencia internacional sugiere que los gobiernos sufren la desconfianza por parte de sus ciudadanos después de un acuerdo de paz con respecto a sus políticas y objetivos futuros. Esto plantea un riesgo para todo el proceso, ya que la población puede permanecer con reservas 
sobre la transparencia de la administración y, en consecuencia, no brindar el apoyo necesario para programas clave como el DDR de los combatientes.

Esta situación, sumada al hecho de que los gobiernos colombianos tradicionalmente registran índices muy bajos de popularidad debido a problemas como la corrupción y el clientelismo, hacen que la implementación de las disposiciones sea aún más difícil. Asimismo, las Fuerzas Militares han tenido los índices más altos de confiabilidad y confianza popular, usualmente por encima del $80 \%$ (El Tiempo, 2015); esta situación podría ser utilizada por el Gobierno para garantizar que los ciudadanos comprendan las obligaciones adquiridas en el acuerdo de paz, enseñar a los excombatientes de las FARC la supremacía del Estado en una democracia y brindar un apoyo crucial para las iniciativas de paz actuales y futuras. Esta propuesta no implica la militarización de la sociedad en absoluto; por el contrario, sugiere la oportunidad de alcanzar una paz sostenible y duradera mediante la reunión de partidos, ciudadanos y esfuerzos en torno a objetivos comunes.

Tercero, la acción unificada, entendida como la "sincronización, coordinación e integración de actividades de entidades gubernamentales y no gubernamentales con operaciones militares para lograr la unidad de esfuerzo" (Ejército Nacional de Colombia, 2016, p. 34), podría ser la base de la construcción de la paz y la implementación de las disposiciones. Durante décadas, los esfuerzos del Gobierno colombiano para aminorar las disparidades políticas y socioeconómicas se han caracterizado por acciones independientes que han dejado vacíos de poder, lo cual ha sido explotado por el crimen organizado y los grupos insurgentes. Esta ausencia de capacidad estatal, junto con la naturaleza del conflicto en Colombia durante los últimos sesenta años, ha llevado al Ejército a asumir mayores responsabilidades para la seguridad pública, además de su papel tradicional en la defensa nacional. Esto ha obligado al Ejército a actuar en nombre de otras instituciones estatales para resolver problemas complejos fuera de su responsabilidad y capacidad.

Una paz firme y sostenible requiere una acción unificada de cada institución del Estado, de manera que aporte en su área de responsabilidad para la construcción de un mejor país. Por lo tanto, el Ejército puede continuar contribuyendo a una acción unificada a través de sus misiones habituales contra las amenazas persistentes y completando tareas como el desminado humanitario del territorio colombiano, la protección de las antiguas áreas dominadas por las FARC, cooperando con programas de sustitución de cultivos, acompañando programas de restitución de tierras y protegiendo a sus nuevos dueños, así como apoyando programas de reforestación en áreas afectadas por el tráfico de drogas. 
En cuarto lugar, apoyar la transformación del Ejército colombiano en una fuerza multimisión que pueda enfrentar amenazas nuevas y persistentes debe ser una prioridad. Esto mejoraría la capacidad del Estado para involucrarse tanto con los desafíos relacionados con las ocho áreas misionales de las Fuerzas Militares ${ }^{20}$-, así como con las amenazas emergentes que deben enfrentarse de manera adecuada y oportuna. Por ejemplo, los casos analizados muestran la alta probabilidad de que haya comercio de armas ilegales de los grupos que se reintegran a la vida civil debido a mecanismos débiles de verificación y custodia; aunque en Colombia la responsabilidad de la custodia de las armas decomisadas recayó exclusivamente en las Naciones Unidas, el Ejército puede utilizar datos de los resultados del proceso para establecer patrones de comercialización, rutas de tráfico y mecanismos de pago.

La transformación del Ejército debe prepararlo para que haga frente a las nuevas fuentes de incertidumbre que podrían ocasionar amenazas futuras. Desastres naturales; un racionamiento repentino de recursos naturales; nuevas enfermedades pandémicas difíciles de tratar; un conflicto interestatal en la región; una escalada terrorista a nivel nacional, regional o mundial; migraciones ilegales a gran escala; el uso de armas nucleares, biológicas, químicas o cibernéticas; o una guerra mundial son solo algunos ejemplos de los desafíos que Colombia podría afrontar y ante los cuales debería estar preparada.

\section{Conclusiones}

Este artículo ha analizado los desafíos actuales al acuerdo de paz colombiano y ha sugerido la importancia que tiene la capacidad estatal en la implementación de las disposiciones. Asimismo, ha ofrecido perspectivas sobre la construcción de paz militar como una opción plausible para garantizar la estabilidad del escenario posterior al acuerdo.

Un acuerdo de paz no significa automáticamente un entorno de estabilidad y gobernanza, y por lo tanto tampoco implica que sea necesario reducir la capacidad de las Fuerzas Militares y los presupuestos de seguridad y defensa. Un análisis de la experiencia internacional sugiere que los acuerdos de paz generalmente incluyen diversas transformaciones de las Fuerzas Militares; sin embargo, una reestructu-

20 Estas son: 1) defensa nacional, 2) seguridad pública, 3) seguridad y convivencia ciudadana, 4) gestión de riesgos, 5) contribución al desarrollo del país, 6) cooperación internacional, 7) protección del medio ambiente y los recursos naturales y 8) gestión, apoyo y desarrollo proyectivo. 
ración inapropiada de los militares puede afectar la capacidad del Estado para enfrentar las amenazas persistentes y emergentes en un escenario de posacuerdo.

Cuando se trata de escenarios complejos posteriores al acuerdo, con restricciones tales como la falta de fondos, tiempo, apoyo y credibilidad, el uso del Ejército puede ser una opción apropiada para ayudar a la estabilización y la consolidación de la paz. Por lo tanto, es importante fortalecer las relaciones cívico-militares y continuar con la transformación en marcha del Ejército Nacional, de manera que se empleen las capacidades de las Fuerzas Militares para descifrar las fuentes de incertidumbre que pueden poner en peligro los logros y los intereses nacionales.

Las experiencias internacionales deben ponderarse en función de las cualidades específicas de los países para identificar los contextos y las particularidades correspondientes. Sin embargo, es necesario reconocer que no hay fórmulas mágicas al comprometerse en la consolidación de la paz; es vital pensar estratégicamente y proponer objetivos realistas en cuanto a alcance, tiempo y costo, así como apoyar un trabajo coordinado que garantice un desarrollo sostenible.

\section{Referencias}

Alto Comisionado para la Paz. (2016). Acuerdo final para la terminación del conflicto y la construcción de una paz estable y duradera [documento en línea]. Recuperado de http://www.altocomisionadoparalapaz.gov.co/procesos-y-conversaciones/Documentos\%20compartidos/24-11-2016NuevoAcuerdoFinal.pdf

Álvarez, E. (2016). Disidencias de las FARC: ¿Por qué lo hacen? ¿Son peligrosas? Recuperado de http://www.ideaspaz.org/publications/posts/1432

Autesserre, S. (2010). The trouble with the Congo: Local violence and the failure of international peacebuilding. Cambridge University Press.

Ávila, A. (2016). La realidad sobre las disidencias de las FARC. Recuperado de http://caracol.com.co/ radio/2016/12/12/politica/1481566427_814038.html

Aya, M. (2017). El proceso de paz en Colombia: Dos pasos adelante, un paso atrás. Estudios Internacionales, 49 (187), 163-179. https://doi.org/10.5354/0719-3769.2017.47034

Bercovitch, J. \& DeRouen, K. (2008). Enduring internal rivalries: A new framework for the study of civil war. Journal of Peace Research, 45 (1), 55-74.

Bourgois, P. (2001). The power of violence in war and peace: Post-Cold War lessons from El Salvador. Ethnography, 2 (1), 5-34. https://doi.org/10.1177/14661380122230803

Castaño, D. (3 de enero de 2017). Deuda externa de Colombia es del 41 \% del PIB. El Colombiano. Recuperado de http://www.elcolombiano.com/negocios/la-deuda-externa-del-gobierno-subio4-64-en-un-ano-emisor-KJ5694862

Deas, M. (2 de octubre de 2012). Ojalá los pesimistas no tengan razón, pero es saludable que se expresen. Revista Credencial. Recuperado de http://www.revistacredencial.com/credencial/ 
noticia/actualidad/portada-malcolm-deas-ojala-los-pesimistas-no-tengan-razon-pero-es-saludable-que

Díaz, C., Fernández, C. \& Rodríguez, J. (2013). Doce miradas del conflicto colombiano [Colección Electrónica, 2]. Madrid: Instituto de Estudios Internacionales y Europeos Francisco de Vitoria. Recuperado de http://perso.unifr.ch/derechopenal/assets/files/obrasportales/op_20130808_01.pdf

Dinero. (4 de junio de 2018). Deuda externa de Colombia en enero de 2018. Recuperado de https:// www.dinero.com/economia/articulo/deuda-externa-de-colombia-en-enero-de-2018/257090

Ejército Nacional de Colombia. (2016). MFE 3-0 Operaciones. Bogotá, D. C.: Ejército Nacional de Colombia. Recuperado de https://www.ejercito.mil.co/?idcategoria=407102\&download=Y

El Nuevo Herald. (5 de marzo de 2017). Colombia preocupada por aumento de cultivos de coca y producción de cocaína. Recuperado de http://www.elnuevoherald.com/noticias/mundo/america-latina/colombia-es/article136607578.html

El Pais. (2 de marzo de 2017). Junta internacional alerta sobre aumento de cultivos de coca en el país entre 2014 y 2015. Recuperado de http://www.elpais.com.co/judicial/junta-internacional-alerta-sobre-aumento-de-cultivos-de-coca-en-el-pais-entre-2014-y-2015.html

El Tiempo. (6 de marzo de 2015). Fuerzas Armadas, con imagen favorable alta, según encuesta. Recuperado de http://www.eltiempo.com/politica/gobierno/fuerzas-armadas-con-imagen-favorable-alta-segun-encuesta/15354035

Escobar, M. (20 de febrero de 2017). Retrasos en las zonas veredales inquietan a misión de ONU. El Colombiano. Recuperado de http://www.elcolombiano.com/colombia/acuerdos-de-gobierno-y-FARC/retrasos-en-zonas-veredales-de-concentracion-de-las-FARC-inquietan-a-laonu-KC5985396

Fernández-Osorio, A. (2016). 2008 Russian Military Reform: An adequate response to global threats and challenges of the Twenty-First Century? Rev. Cient. Gen. José María Córdova, 14 (17), 41-82.

Frieden, S. E. und. (2002). Fragile peace: State failure, violence and development in crisis regions. London and New York: Zed Books.

Ginty, R. M. (2010). No war, no peace: Why so many peace processes fail to deliver peace. International Politics, 47(2), 145-162. https://doi.org/10.1057/ip.2010.4

Gómez, M. (25 de febrero de 2017). El miércoles, las FARC comienzan su adiós a las armas-Proceso de paz. El Tiempo. Recuperado de http://www.eltiempo.com/politica/proceso-de-paz/esta-semanacomienza-desarme-de-las-FARC/16828868

Hartzell, C. (2010). Crafting peace: Power-sharing institutions and the negotiated settlement of civil wars. University Park: Penn State Press.

Holiday, D. \& Stanley, W. (1993). Building the peace: Preliminary lessons from El Salvador. Journal of International Affairs, 46 (2), 415-438.

Iglesias, E. S. \& Jiménez, V. S. (2018). La paz y la solución al problema del campo en Colombia: un análisis comparado entre el Acuerdo de Paz y el Plan Nacional de Desarrollo. Araucaria, 20 (39), 365-387. Recuperado de https://revistascientificas.us.es/index.php/araucaria/article/view/4912

Fondo Monetario Internacional. (2017). World Economic Outlook Database. International Monetary Fund. Recuperado de https://www.imf.org 
Joshi, M., Melander, E. \& Quinn, J. M. (2017). Sequencing the peace: How the order of peace agreement implementation can reduce the destabilizing effects of post-accord elections. Journal of Conflict Resolution, 61 (1), 4-28. https://doi.org/10.1177/0022002715576573

Joshi, M. \& Wallensteen, P. (eds.). (2018). Understanding quality peace: Peacebuilding after Civil War. London: Routledge.

Kroc Institute for International Peace Studies. (2018). Peace Accords Matrix. Recuperado de https:// peaceaccords.nd.edu/about

Meacham, C., Farah, D. \& Lamb, R. (2014). Colombia: Peace and stability in the post-conflict era. Washington, D. C.: Center for Strategic and International Studies. Recuperado de http://csis. org/files/publication/140304_Meacham_Colombia_Web.pdf

Merriam-Webster. (2017). esprit de corps. Recuperado de http://www.merriam-webster.com/dictionary/esprit $\% 20 \mathrm{de} \% 20$ corps

Ministerio de Defensa Nacional. (2016). Directiva 015. Ministerio de Defensa Nacional [documento en línea]. Recuperado de https:/www.mindefensa.gov.co/irj/go/km/docs/Mindefensa/ Documentos/descargas/Prensa/Documentos/dir_15_2016.pdf

Molano, A. (2015). El posconflicto en Colombia: Reflexiones y propuestas para recorrer la transición. Bogotá, D. C.: Instituto de Ciencia Política Hernán Echavarría Olózaga y Fundación Konrad Adenauer Colombia. Recuperado de http://www.icpcolombia.org/dev/wp-content/ uploads/2015/12/posconflicto.pdf

Moreno, J. D. (2017). Paz, memoria y verdad en El Salvador: Experiencias y lecciones para la Colombia del posacuerdo. Análisis Político, 30 (90), 175-193. https://doi.org/10.15446/anpol. v30n90.68560

Paris, R. (2004). At war's end: Building peace after civil conflict. Cambridge: Cambridge University Press.

Patiño, C. (2013). Guerra y construcción del Estado en Colombia 1810-2010. Bogotá, D. C.: Penguin Random House.

Rocha, A. (2011). State building for peace: A new paradigm for international engagement in post-conflict fragile states? Third World Quarterly, 32 (10), 1715-1736.

Roeder, P. G. \& Rothchild, D. S. (2005). Sustainable peace: Power and democracy after civil wars. Cornell University Press.

Sáenz, J. (15 de junio de 2017). ¿Cuánto costará la paz con las FARC? El Espectador. Recuperado de https://colombia2020.elespectador.com/politica/la-paz-le-costara-colombia-130-billones

Semana. (2017). ¿Los retrasos en la construcción de las zonas veredales demorará el inicio del desarme de las FARC? [Video]. Recuperado de http://www.semana.com/nacion/multimedia/retrasos-en-la-construccion-en-las-zonas-veredales-amenazan-el-proceso-de-paz/516486

Stockholm International Peace Research Institute [Sipri]. (2016). SIPRI Military Expenditure Database. Stockholm International Peace Research Institute. Recuperado de https://www.sipri. org/databases/milex

Taydas, Z., Peksen, D. \& James, P. (2010). Why do civil wars occur? Understanding the Importance of Institutional Quality. Civil Wars, 12 (3), 195-217.

Vacas Fernández, F. (2018). El conflicto de Colombia y los acuerdos de paz en perspectiva internacional. Bogotá, D. C.: Ediciones Doctrina y Ley. 
Vargas, A. (2010). Las Fuerzas Armadas en el conflicto colombiano: antecedentes y perspectivas. Bogotá, D. C.: La Carreta Editores.

Vargas, J. (2018). Enfoque de género en el acuerdo de paz entre el Gobierno colombiano y las FARCEP: Transiciones necesarias para su implementación. Araucaria, 20(39), 390-414. Recuperado de https://revistascientificas.us.es/index.php/araucaria/article/view/4913

Walter, B. (1999). Designing transitions from civil war: Demobilization, democratization, and commitments to peace. International Security, 24(1), 127-155. 